\title{
Environmental risk assessment of advanced therapies containing genetically modified organisms in the EU
}

\author{
Rhys Whomsley ${ }^{1}$, Victoria Palmi Reig ${ }^{1}$, and Ana Hidalgo-Simon ${ }^{1}$ \\ ${ }^{1}$ European Medicines Agency
}

June 22, 2020

\begin{abstract}
Gene therapy medicinal products have the potential to provide curative treatment to many diseases with current limited therapeutic options. As advanced therapy medicinal products (ATMPs), these therapies undergo a centralised, single European Union authorisation by the European Medicines Agency, but the risks, and potential harm to the environment and population at large are weighted in each application, and different interpretations at national level exist. A streamlined procedure is now in place to facilitate a consistent approach for the assessment of the environmental risks of medicines containing genetically modified organisms. This article provides an overview of basic requirements across the EU, an overview of the new streamlined process and discusses available guidance for developers. All these initiatives are aimed to remove hurdles for ATMP developers and facilitate faster access to patients.
\end{abstract}

\section{Introduction}

Advanced Therapy Medicinal Products (ATMPs) are an umbrella term that includes gene therapy, cellbased, tissue engineered products and combined ATMPs (cATMPs) $\left[{ }^{1},{ }^{2}\right]$. In regulatory terms, gene therapy medicinal products (GTMPs) are medicines with an active substance that contain or consist of a recombinant nucleic acid. That nucleic acid is present with the intention of regulating, repairing, replacing, adding, or deleting a genetic sequence in the patient, and whose therapeutic, diagnostic, or prophylactic effect relates either directly or indirectly through a protein it expresses. For regulatory purposes vaccines against infectious diseases are not included as ATMPs $\left[{ }^{3}\right]$.

When medicines developers apply for a marketing authorisation for a human medicinal product, they are required to submit an environmental risk assessment (ERA). The genetically modified organism, or GMO, contained in GTMPs is the focus of the ERA of these medicines.

The ERA for GMOs is based on quality, preclinical and clinical data. The process consists of (1) hazard identification, (2) hazard characterization, (3) assessment of likelihood, and (4) risk estimation. These data together with the likelihood of the adverse event occurring and the consequences of such an event, constitute the ERA $\left[{ }^{4}\right]$. In addition to the risk to the environment per se, the ERA also considers the potential harmful effects on third parties exposed to the GMO-containing ATMP, such as medical personnel handling the product $\left.{ }^{4}\right]$.

GTMPs, and all ATMPs, fall under the mandatory scope of the centralized procedure, which means that a single central application to the European Medicines Agency (EMA) will achieve marketing authorisation for the whole of the EU. During the assessment that leads to a marketing authorisation, the ERA is reviewed by two committees; the Committee for Advanced Therapies (CAT) and the Committee for Human Medicinal Products (CHMP). In addition, and in accordance with the legislation, the ERA is also subject to consultation by the competent authority appointed to be responsible for GMO dossiers at national level. The fact that 
different countries in the EU have different requirements, and sometimes involve additional bodies creates additional complexity for developers.

Developers of GTMPs are also required to submit a GMO-specific ERA prior to conduct of clinical trials. This is dependent on the country in which the trial is to be performed and the level of containment considered necessary for the product in the conduct of the trial. Under EU legislation, clinical trials with human products containing or consisting of GMOs can be performed under contained use (CU) $\left[{ }^{5}\right]$ or deliberate release (DR) $\left.{ }^{6}\right]$ legislation (see glossary 1).

Complications arise because these directives, implemented into the national legislations, are not uniformly applied or interpreted by member states (see Table 1 for details country by country). It is generally only for DR procedures that a formal ERA and a detailed technical/scientific description of the GMO are required. There are significant differences between member states in the documentation and procedures required for authorisation of GMO aspects of clinical trials for medicines containing GMO and a public consultation is also required in some countries. The current approach is not ideal for the conduct of multinational clinical trials and has been considered by many an impediment to the effective translation of research findings into clinical applications $\left[{ }^{7},{ }^{8},{ }^{9}\right]$.

This commentary reviews recent initiatives taken by the European Commission towards a common procedure for the ERA review for clinical trials applications for GMO-containing medicinal products, in particular for cell-based therapies and some viral vector based GTMPs where most experience is available, and outlines steps taken by the EMA to streamline the assessment procedures for GMO-containing products for marketing authorisation applications. Although chiefly focused on GTMPs, these approaches will also be relevant for some products other than GTMPs such as GMO-containing vaccines.

\section{Clinical Trial Applications}

In most circumstances, ERAs are required only for clinical trials conducted under the deliberate release legislative framework. In Table 1, the legislative framework under which clinical trials with GMOs may be conducted in the different member states are listed together with the sequence of authorisations required $\left.{ }^{8}\right]$.

Some countries only allow the conduct of GMO clinical trials under one procedure or the other, however, other countries will consider either procedure based on a case-by-case evaluation. The content and format requirements of the DR procedure are broadly harmonised across EU countries through the standardised use of the summary notification information format for notifications concerning the deliberate release into the environment of genetically modified organisms for purposes other than for placing on the market, and ERA templates $\left[{ }^{7},{ }^{10}\right]$. Contained use dossiers do not require an ERA but focus on a description of the GMO facilities, the GMO handling, waste management and protection of workers with the format differing from country to country $\left[{ }^{7}\right]$.

The sequence of steps required in the review of the GMO dossier and the clinical trial application and the identity of national competent authorities and advisory bodies for both the contained use and deliberate release procedure varies according to each country. For trials conducted under the deliberate release approach a public consultation is generally required and the summary notification information dossier becomes publicly available on the EU GMO register. However, some clinical trials conducted under contained use may also require a public consultation if the member state considers it appropriate $\left[{ }^{7}\right]$. These differences in interpretation of environmental and biosafety aspects for GMO regulatory procedures across different countries have led to divergent opinions in classifications regarding the clinical use of the GMO medicine and adds to the complexity of conducting multinational clinical trials with GMOs $\left[{ }^{11}\right]$.

The new Clinical Trial Regulation $\left[{ }^{12}\right]$ will not address many of the issues associated with GMO-containing clinical trials. Currently, some member States require authorisation under the GMO framework before the Clinical trial application can be submitted (see Table 1). When the regulation becomes applicable, the authorisation under the GMO framework can no longer be a pre-requisite for a valid clinical trial authorisation application but it will still be required before the clinical trial can start $\left[{ }^{13}\right]$. 
To overcome these hurdles, the national competent authorities and the European Commission have recently updated and published good practice documents and common application forms concerning the conduct of clinical trials with human medicinal products consisting of or containing GMOs $\left[{ }^{14},{ }^{15},{ }^{16},{ }^{17},{ }^{18}\right]$. These documents aim to facilitate the conduct of clinical trials by achieving a degree of harmonisation and clarifying the requirements for clinical trial applications based on the existing legislation. They have also introduced, for certain categories of investigational medicinal products, a "specific ERA" on the basis that they are highly unlikely to pose a risk to the environment or to public health.

The common application form for viral vectors contained in investigational medicinal products for human use are applicable to medicinal products for human use that contain or consist of viral vectors $\left.{ }^{14}\right]$. Section 2 of the common application form concerns the information relating to the investigational medicinal product (parental virus, genetic modification and clinical vector) while the ERA (to be completed for submissions under deliberate release, or for all submissions for clinical trials to be performed in Italy) is in Section 5. A GMO-specific ERA is required (Annex II of Directive 2001/18/EC) for all applications under the deliberate release framework, however, two categories of products, for which specific common application forms apply $\left[{ }^{15},{ }^{16}\right]$ qualify for "specific ERAs".

\section{Specific ERA}

The most important elements to consider for the ERA of GTMPs are the type of viral vector and whether the viral vector is constituted into genetically modified cells. The key to environmental risk normally focuses on the potential for the presence or generation of an infective viral agent $\left[{ }^{11}\right]$.

Investigational medicinal product'IMPs for human use that contain or consist of Adeno-associated (AAV) vectors. Developers are eligible for specific ERAs if the applicant demonstrates:

i) absence of formation of replication competent virus;

ii) that the transgene is not harmful.

AAVs have not been associated with any pathogenic disease in humans or animals and are unable to replicate unless the cell is co-infected with a helper virus. Therefore, the only mechanism by which there could be mobilisation is that the same cell was infected simultaneously with the clinical vector, a wild-type AAV virus and a helper virus (triple infection). The likelihood of simultaneous triple infection can be considered very low. In addition, most of the genome of the wild-type virus is removed in vectors used in clinical practice, except for the inverted terminal repeats. The recombined particles would have the rep and cap genes encoding for proteins required for replication and capsid formation but they would still be replicationdefective (as the wild-type virus). In view of these factors and provided that the control measures described by the applicant are implemented, the overall risks for human health and the environment can be considered negligible $\left[{ }^{15},{ }^{17}\right]$.

Human cells genetically modified by means of retro/lentiviral vectors. Human cells cannot proliferate in the environment as they can only survive inside the human body or under in vitro culture conditions. It follows that, when the IMP consist of human cells genetically modified by means of retro/lentiviral vectors, the risks to the environment and public health are mainly linked to the potential for the viral elements to remobilize, with or without recombination with other elements, to release infective virus into the environment $\left[{ }^{16},{ }^{18}\right]$.

Developers of IMPs consisting of human cells genetically modified by means of retro/lentiviral vectors are eligible for the specific ERA described in the Annex of the Good Practice document on the assessment of GMO-related aspects in the context of clinical trials with human cells genetically modified by means of retro/lentiviral vectors if the applicant demonstrates;

i) the absence of formation of replication competent virus;

ii) the absence of residual infectious viral vector particles in the transduced cells.

Under these circumstances, no risks to the environment or animal health can be identified. Provided that 
the control measures described by the applicant are implemented, the overall risks for third parties exposed to the GMO-containing medicinal product are also considered negligible.

The good practice document specifies that retroviral vectors means murine gamma-retroviral vectors and that lentiviral vectors are those derived from human cells transduced with lentiviral vectors derived from HIV virus. In case of lentiviral vectors derived from other viruses, developers should perform a risk assessment and contact the relevant competent authority.

\section{Marketing Authorisation Applications}

In the EU, marketing authorisation of GTMPs falls under the mandatory scope of the central authorisation procedure, through submission to, and review by, the EMA and its committees. The CAT is responsible for the primary evaluation of ATMP marketing authorisation applications for the CHMP. Marketing authorisation of GTMPs requires, as for all medicinal products, that the applicant demonstrates the quality, safety and the efficacy in patients. The data submitted by developers of GTMPs in their application for marketing authorisation must include a range of information, including the way the medicine is manufactured, its effects in laboratory studies, benefits and side effects observed in patients, and how risks will be managed, as well as the proposed information to be provided to patients and doctors. It is also important to ensure that environmental risks from innovative medicines are understood and controlled and this is discussed in the ERA. The objective of an ERA is to identify and evaluate any potential adverse effects of the GMO, either direct or indirect, immediate or delayed, on human health and the environment. Importantly, the ERA should be conducted with a view to evaluate if there is a need for risk management, and if so, the most appropriate methods to use. Two guidelines have been issued by the EMA on the expected content for the ERA for GTMP and/or medicinal products containing GMOs $\left[{ }^{4},{ }^{19}\right]$.

At the same time, in line with the regulatory science strategy of the Agency and to support the translation of ATMPs into patient treatments, the EMA has recognised the need to streamline interactions with ERA Competent authorities during the assessment of the ERA.

The improvements in this field have been made through complementing the guidelines with detailed recommendations for dossier submissions in the 'European Medicines Agency pre-authorisation procedural advice for users of the centralised procedure' $\left[{ }^{20}\right]$.which provide assistance with early planning, method development and evaluation; and updating procedures to facilitate a consistent approach for the assessment of the environmental risks of medicines containing GMO $\left[{ }^{21}\right]$.

The common application forms developed for the assessment of GMO aspects in the context of clinical trials with IMPDs already adapt the technical requirements to the characteristic of human cells genetically modified by means of retro/lentiviral vectors or medicinal products that contain AAV vectors. To reduce administrative burdens, the technical requirements at the time of filing, marketing authorisation applications are built on the same common application forms used for clinical trial approval. The medicinal products eligible for a "specific ERA" in the context of clinical trial applications are also eligible for this type of ERA at marketing authorisation applications.

In addition, to deliver a contribution that is fit for purpose in view of the sharp increase of the number of ATMPs in development and expected marketing authorisation applications involving GMOs, the consultation process with national competent authorities responsible for environmental risks is now focused on the questions/aspects identified by the rapporteur, in particular on the risk minimisation measures.

\section{Consultation with GMO-CA during the Marketing application}

Overall, the assessment of a new medicine is completed within 210 active review days. This active evaluation time is the time spent by EMA experts to evaluate the evidence provided by the applicant in support of marketing authorisation applications. This time is interrupted by one or two 'clock-stops' during which the applicant prepares the answers to any questions raised by the CAT. The duration of a clock-stop depends on how long the applicant thinks it will take to respond. 
For each new medicine, two CAT members- known as rapporteur and co-rapporteur - from different countries are appointed to lead the assessment.

Experts with specialised scientific knowledge are often consulted during the evaluation to enrich the scientific discussion and for medicines containing a GMO, the GMO Competent authorities responsible for environmental risks in each Member State must be consulted.

This consultation is handled, in the same way that all CAT members contribute actively to the evaluation process, by reviewing the assessments made by the rapporteurs, providing comments and identifying additional questions to be addressed by the applicant.

As a general principle, the consultation with the environmental authorities, shown in figure 1, only takes place during the 1st phase of the marketing authorisation applications evaluation and the focus is in advising the CAT on the best measures to prevent dissemination into the environment.

Although the ERA is reviewed together with the rest of the dossier by EMA, the ERA must also be presented as a self-standing document to allow distribution to Environmental Competent Authorities as part of the necessary consultation. All parties involved in the evaluation of an application are required to respect confidentiality in relation to all data contained in the dossier and assessment reports relating to the product. The information will only be made available to the public after the approval of the marketing authorisation as part of the European Public Assessment Report.

In addition, it should be pointed that the consultation procedure must be conducted in accordance with the framework and the deadlines of the medicine evaluation procedure (e.g. deadlines and consultation procedures under GMO framework are not applicable).

It is important that developers identify prior to dossier submission if there is any need for control measures during reconstitution, handling and administration, personal protective equipment, decontamination or cleaning measures after administration or in case of accidental spilling, elimination of left-overs or waste management, recommendations to patients to prevent dissemination or warnings regarding donation of blood, cells tissues or organs including realistic durations for these precautions. The common application forms for ERAs and good practice documents provide a very comprehensive guidance to build the environmental risk assessment.

While it is a regulatory requirement to assess thoroughly all the potential risks and define measures to minimise any potential for exposure, the EMA also expects that recommendations and warnings are proportionate to the actual hazards posed and recommends avoiding overcomplicated procedures or unnecessary protective equipment. At the same time, even when the ERA may be more straightforward, it is also expected that the justifications to reach certain conclusion must be provided and the absence of warnings discussed.

\section{Discussion}

GTMPs are a rapidly growing therapeutic modality with the potential for curative treatment of many genetic diseases unresponsive to conventional therapies $\left.{ }^{9}\right]$. In addition to genetic defects, several other conditions that that currently have limited, or no effective therapeutic options can be cured with gene therapy. However, due to their complexity, GTMPs developers have faced major regulatory challenges during assessment of clinical trial applications and marketing authorisation applications $\left[{ }^{1},{ }^{2}\right]$ and these include aspects related to environmental risk assessment. While it is acknowledged that there may be genuine environmental risks from genetically modified viruses and that these must be rigorously assessed, there are categories of products consisting of or containing genetically modified viruses which have been shown to have negligible environmental risk under their conditions of use.

The introduction of common application forms and good practice documents have been an important step in the simplification of the clinical trial application procedures for medicines consisting of or containing GMOs. Although the differences in the legislative frameworks and authorisation procedures between member states 
remain, the common application forms harmonise the common information needed in the applications and provide information on the additional country-specific requirements.

All marketing authorisation applications for GTMPs need to include a GMO-specific ERA in the submission. This is the case even if all clinical trials have been performed under the CU legislative framework for which an ERA is not required or if the trials were conducted outside of the EU, where different GMO requirements exist. An important advance for GMTPs is the introduction of specific ERAs for certain classes of products (AAVs and human cells with retroviruses/lentiviruses). The latter includes chimeric antigen receptor Tcells (CAR-T) which are one of the most promising subclasses of GTMPs in the field of cellular cancer

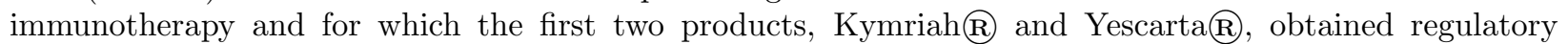
approval in the EU in 2018. The introduction of the common application forms simplifies the regulatory pathway for subsequent similar products in development with regards to GMO-related issues for both clinical trial applications and marketing authorisation applications. Applicants eligible for specific ERAs for clinical trial applications should also submit specific ERAs at marketing authorisation applications.

The two EMA guidelines on the ERA for GTMPs and GMOs should be consulted together with the recently updated EMA pre-authorisation procedural advice for medicinal product containing or consisting of GMOs which refers to the appropriate common application forms and good practice documents. The recent revision of the SOP on the Consultation of environmental competent authorities on genetically modified organisms with respect to environmental risk assessment for medicinal products for human use will streamline the assessment procedure and bring clarity to both developers and regulators. In addition, the Agency will monitor and continue to streamline this process as experience grows and feedback is received.

Finally, although the focus of this article has been the ERA of GTMPs, the same principles are also applicable to the development of vaccine candidates which involve genetic modification and are therefore subject to GMO legislation. In light of current disease outbreaks (e.g. COVID-19 pandemic, Ebola) the European Commission, together with the Member States and the European Medicines Agency, will make the greatest use of existing flexibilities in the EU's regulatory framework to accelerate the authorisation and availability of successful vaccines against COVID-19. Among other initiatives, the EC has put forward a proposal to provide temporary derogations from certain provisions of the GMO legislation to speed up clinical trials of COVID-19 vaccines containing genetically modified organisms $\left[{ }^{22}\right]$. The proposed Regulation will permit clinical trials with GMO medicinal products against COVID-19 to start in the absence of an ERA and explicit consent under GMO legislation, but introduces limitations to minimise environmental exposure. The massive international efforts to develop a vaccine for COVID-19, will provide candidates to be tested over the second half of 2020, leading to a practical trial of how these simplifications of GMO processes in the EU will work in practice.

\section{Conclusions}

A streamlined procedure is now in place in the EU to facilitate a consistent approach for the assessment of the environmental risks of medicines containing GMOs. Different countries have different approaches to this issue, but the risks, and potential harms to the environment and population at large are weighted in each application.

Following available guidance is essential to avoid questions and delays at approval time and help the preparation of the environmental risk assessment necessary in all marketing applications.

With these initiatives regulators are answering the calls to facilitate research and assessment of the environmental risk aspects of medicines containing GMOs in Europe.

\section{Glossary}

\begin{tabular}{ll}
\hline ATMP & Advanced therapy medicinal product \\
\hline CAT & Committee for advanced therapies \\
CHMP & Committee for medicinal products for human use
\end{tabular}




\begin{tabular}{ll}
\hline ATMP & Advanced therapy medicinal product \\
\hline ERA & Environmental risk assessment \\
GTMP & Gene therapy medicinal product \\
MAA & Marketing authorisation application \\
DR & Deliberate Release. Any intentional introduction into the environment for which no specific containment measure \\
GMO & Genetically Modified Organisms \\
CU & Contained Use. Activity for which specific containment measures are used to limit their contact with, and to prov \\
CT & Clinical Trial \\
AAV & Adeno associated virus \\
IMP & Investigational medicinal product \\
\hline
\end{tabular}

\section{Disclaimer}

The views expressed in this article are the personal views of the authors and may not be understood or quoted as being made on behalf of or reflecting the position of the European Medicines Agency or one of its committees or working parties.

\section{Acknowledgments}

We thank Drs Enrica Alteri, Milton Bonelli, Veronika Jekerle, Patrick Celis, Thomas Castelnovo and Ana Rodriguez Sanchez Beato for their valuable suggestions. We are also very grateful to Charlotte Hallin for her invaluable editorial and logistical support.

\section{Tables}

Table 1: Authorisation procedures for clinical trials with human medicinal products containing GMOs in EU member states

Process for authorisation of Clinical Trials

Contained use or Deliberate release

Authorisation under the clinical trials and under the GMO framework are not linked

Czechia, Cyprus, Finland, France, Italy, Portugal
Process for authorisation of Clinical

Contained use or Deliberate release

Authorisation of GMO aspects is re Bulgaria

${ }^{1}$ The administration of the product to the clinical trial subject requires an approval under special regulations for gene therapy only.

Figure 1: Consultation process with environmental competent authorities with respect to environmental risk assessment for medicinal products for human use during assessment of marketing authorisation applications

1. Carvalho M, Martins AP, Sepodes B. Hurdles in gene therapy regulatory approval: a retrospective analysis of European Marketing Authorization Applications. Drug Discov Today . 2019;24(3):823-828. doi:10.1016/j.drudis.2018.12.007

2. Iglesias-López C, Agustí A, Obach M, Vallano A. Regulatory Framework for Advanced Therapy Medicinal Products in Europe and United States.Front Pharmacol . 2019;10:921. doi:10.3389/fphar.2019.00921

3. Commission Directive 2009/120/EC of 14 September 2009 Amending Directive 2001/83/EC of the European Parliament and of the Council on the Community Code Relating to Medicinal Products for Human Use as Regards Advanced Therapy Medicinal Products (Text with EEA Relevance) . Vol OJ L.; 2009. Accessed June 11, 2020. http://data.europa.eu/eli/dir/2009/120/oj/eng

4. European Medicines Agency. EMA Corr Guideline on environmental risk assessments for medicinal products consisting of, or containing, genetically modified organisms. 11 December 2006. Published Decem- 
ber 2006. Accessed June 11, 2020. https://www.ema.europa.eu/en/documents/scientific-guideline/guidelineenvironmental-risk-assessments-medicinal-products-consisting-containing-genetically_en.pdf

5. Directive 2009/41/EC of the European Parliament and of the Council of 6 May 2009 on the contained use of genetically modified micro-organisms (Recast)Text with EEA relevance. :23.

6. Directive 2001/18/EC of the European Parliament and of the Council of 12 March 2001 on the Deliberate Release into the Environment of Genetically Modified Organisms and Repealing Council Directive 90/220/EEC - Commission Declaration. Vol OJ L.; 2001. Accessed June 11, 2020. http://data.europa.eu/eli/dir/2001/18/oj/eng

7. Kauffmann F, Van Damme P, Leroux-Roels G, et al. Clinical trials with GMO-containing vaccines in Europe: Status and regulatory framework. Vaccine . 2019;37(42):6144-6153. doi:10.1016/j.vaccine.2019.08.018

8. ARM, EFPIA, EBE, EuropaBio. Position paper: Possible solutions to improve the European regulatory procedures for clinical trials with Advanced Therapy Medicinal Products consisting of or containing Genetically Modified Organisms. Published September 27, 2017. Accessed May 28, 2020. https://www.ebe-biopharma.eu/wp-content/uploads/2017/09/Position_paper_ARM_EFPIA_EBE_EuropaBio_27Sept17_longversion.pdf

9. Ten Ham RMT, Hövels AM, Klungel OH, Leufkens HGM, Broekmans AW, Hoekman J. Development and Regulation of Gene and Cell-Based Therapies in Europe: A Quantification and Reflection. Trends Pharmacol Sci . 2020;41(2):67-71. doi:10.1016/j.tips.2019.11.007

10. 2002/813/EC: Council Decision of 3 October 2002 Establishing, Pursuant to Directive 2001/18/EC of the European Parliament and of the Council, the Summary Notification Information Format for Notifications Concerning the Deliberate Release into the Environment of Genetically Modified Organisms for Purposes Other than for Placing on the Market . Vol 280.; 2002. Accessed June 16, 2020. http://data.europa.eu/eli/dec/2002/813/oj/eng

11. Bachtarzi H, Farries T. The Genetically Modified Organism Medicinal Framework in Europe, United States, and Japan: Underlying Scientific Principles and Considerations Toward the Development of Gene Therapy and Genetically Modified Cell-Based Products. Hum Gene Ther Clin Dev . 2019;30(3):114-128. doi:10.1089/humc.2019.042

12. Regulation (EU) No 536/2014 of the European Parliament and of the Council of 16 April 2014 on Clinical Trials on Medicinal Products for Human Use, and Repealing Directive 2001/20/EC Text with EEA Relevance. Vol 158.; 2014. Accessed June 16, 2020. http://data.europa.eu/eli/reg/2014/536/oj/eng

13. Medicinal products for human use containing or consisting of GMOs: interplay between the EU legislation on medicinal products and GMOs - frequently asked questions. Accessed June 16, 2020. https://ec.europa.eu/health/sites/health/files/files/advtherapies/docs/gmcells_qa_en.pdf

14. Common application form for viral vectors contained in investigational medicinal products for human use. Accessed June 11, 2020. https://ec.europa.eu/health/sites/health/files/files/advtherapies/docs/vvs_caf_en.pdf

15. Common application form for investigational medicinal products for human use that contain or consist of AAV vectors. Accessed June 11, 2020. https://ec.europa.eu/health/sites/health/files/files/advtherapies/docs/aavs_caf_en.pdf

16. Common Application form for clinical research with human cells genetically modified by means of retro/lentiviral vectors. Accessed June 11, 2020 . https://ec.europa.eu/health/sites/health/files/files/advtherapies/docs/gmcells_caf_en.pdf

17. Good Practice on the assessment of GMO related aspects in the context of clinical trials with AAV clinical vectors. Accessed June 11, 2020. https://ec.europa.eu/health/sites/health/files/files/advtherapies/docs/aavs_gp_en.pdf 
18. Good Practice on the assessment of GMO-related aspects in the context of clinical trials with human cells genetically modified by means of retro/lentiviral vectors. Accessed June 16, 2020. https://ec.europa.eu/health/sites/health/files/files/advtherapies/docs/gmcells_gp_en.pdf

19. European Medicines Agency. Guideline on Scientific Requirements for the Environmental Risk Assessment of Gene Therapy Medicinal Products. https://www.ema.europa.eu/en/documents/scientificguideline/guideline-scientific-requirements-environmental-risk-assessment-gene-therapy-medicinal-

products_en.pdf. Published online 2008:14.

20. European Medicines Agency. EMA pre-authorisation guidance. Published September 17, 2018. Accessed June 11, 2020. https://www.ema.europa.eu/en/human-regulatory/marketing-authorisation/preauthorisation-guidance

21. European Medicines Agency. EMA standard-operating-procedure-consultation-environmentalcompetent-authorities-genetically-modified_en.pdf. Published December 2019. Accessed June 11, 2020. https://www.ema.europa.eu/en/documents/sop/standard-operating-procedure-consultation-environmentalcompetent-authorities-genetically-modified_en.pdf

22. European Commission. Press release: Coronavirus: Commission unveils EU vaccines strategy. Published June 17, 2020. Accessed June 17, 2020. https://ec.europa.eu/commission/presscorner/detail/en/ip_20_1103

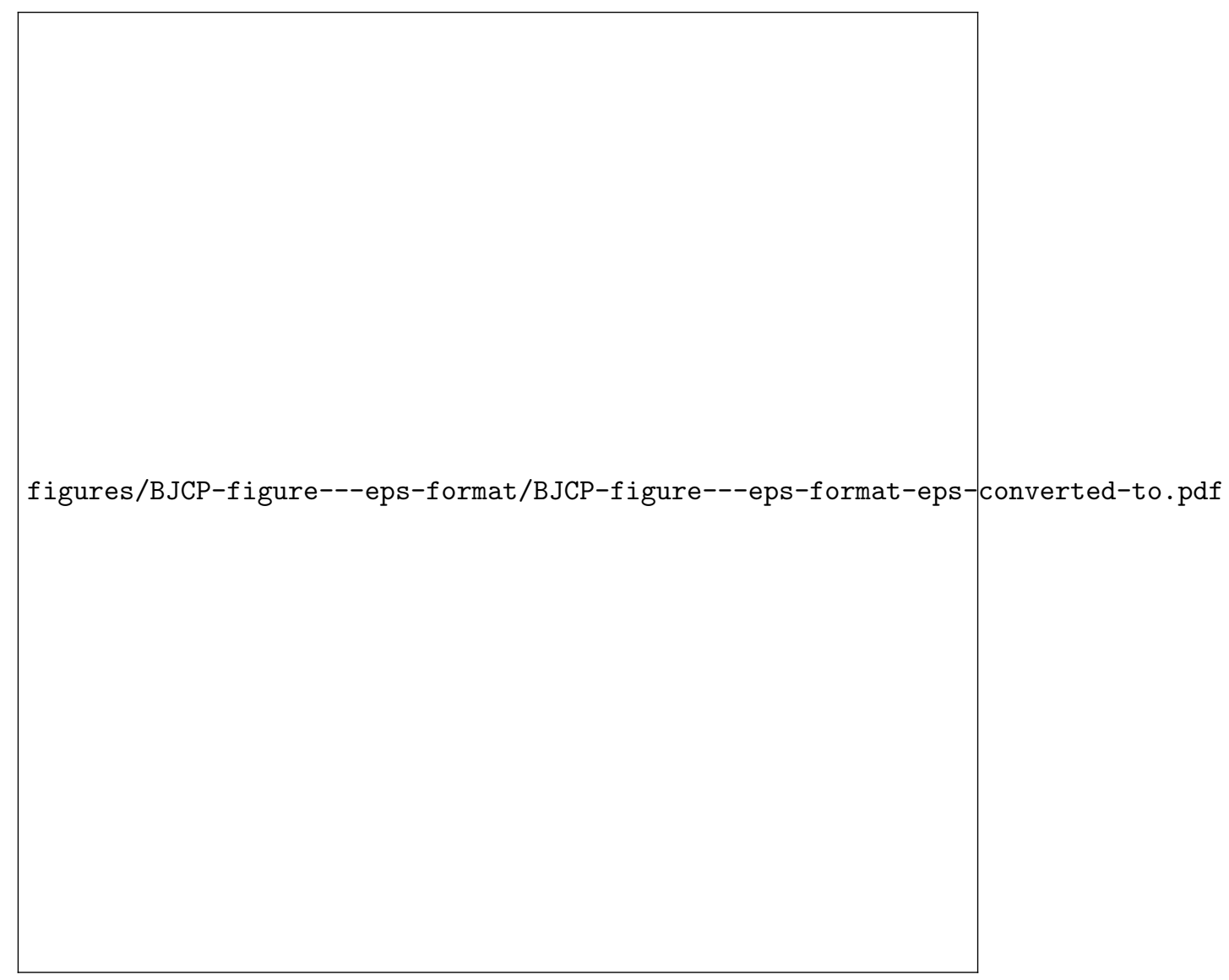

